\title{
Perturbation-Iteration Method for First-Order Differential Equations and Systems
}

\author{
Mehmet Şenol, ${ }^{1}$ İhsan Timuçin Dolapçı, ${ }^{2}$ Yiğit Aksoy, ${ }^{2}$ and Mehmet Pakdemirlii ${ }^{2}$ \\ ${ }^{1}$ Department of Mathematics, Nevsehir University, 50300 Nevsehir, Turkey \\ ${ }^{2}$ Department of Mechanical Engineering, Celal Bayar University, Muradiye, 45140 Manisa, Turkey \\ Correspondence should be addressed to Mehmet Pakdemirli; mpak@cbu.edu.tr
}

Received 16 March 2013; Revised 18 April 2013; Accepted 19 April 2013

Academic Editor: Yisheng Song

Copyright (C) 2013 Mehmet Şenol et al. This is an open access article distributed under the Creative Commons Attribution License, which permits unrestricted use, distribution, and reproduction in any medium, provided the original work is properly cited.

\begin{abstract}
The previously developed new perturbation-iteration algorithm has been applied to differential equation systems for the first time. The iteration algorithm for systems is developed first. The algorithm is tested for a single equation, coupled two equations, and coupled three equations. Solutions are compared with those of variational iteration method and numerical solutions, and a good agreement is found. The method can be applied to differential equation systems with success.
\end{abstract}

\section{Introduction}

Perturbation methods are classical methods which have been used over a century to obtain approximate analytical solutions. The method has been successfully applied to differential equations, integro differential equations, and algebraic equations. Many different perturbation techniques such as the method of multiple scales, the method of averaging, the renormalization method, the Lindstedt-Poincare method, the method of matched asymptotic expansions, and their variants were developed $[1,2]$.

The major limitation of the perturbation methods is the requirement of a small parameter. Sometimes the small parameter may also be artificially introduced into the equations. The solutions therefore have a limited range of validity. Although the solutions are valid for weakly nonlinear problems, they are not admissible usually for strongly nonlinear problems.

Several techniques have been proposed in the literature recently to obtain admissible solutions which do not require small parameter assumption. While a complete review of the attempts is beyond the scope of this work, linearized perturbation method, parameter expanding method, new time transformations as modifications of Lindstedt-Poincare method, and iteration methods can be mentioned as examples [3-14].
Recently, a class of alternative perturbation-iteration algorithms has been proposed. The fundamentals of the algorithms were outlined for the first-order differential equations by Pakdemirli et al. [15]. Several iteration algorithms can be derived by taking different number of terms in the perturbation expansions and different order of correction terms in the Taylor series expansions. The perturbation iteration algorithm is called $\operatorname{PIA}(n, m)$ where $n$ represents the correction terms in the perturbation expansion and $m$ represents the highest order derivative term in the Taylor series. This new method has been successfully implemented to Bratu-Type equations [16]. Solutions obtained by this new method and those obtained by variational iteration method (VIM) were contrasted, and it is shown that while $\operatorname{PIA}(1,1)$ algorithm usually produces identical results with VIM, higher order algorithms $\operatorname{PIA}(n, m)$ produce better results. The new perturbation-iteration technique was applied to nonlinear heat transfer equations by Aksoy et al. [17] very recently. One of the main advantages is that the new method does not require initial assumptions or transformation of the equations to another form. Actually, the techniques were developed first for algebraic equations [18-20] and then adopted to ordinary differential equations [15-17].

In this study, the iteration algorithms for single equations are generalized to arbitrary number of first-order coupled equations. An application of the algorithm to a single 
equation which is a degenerate case is treated first. Then, coupled systems with two and three equations are solved. Solutions are contrasted with available other approximate solutions or numerical solutions. It is found that the method can be effectively applied to differential equation systems as well.

\section{Perturbation-Iteration Algorithm $\operatorname{PIA}(1, m)$}

In this section, a perturbation-iteration algorithm $\operatorname{PIA}(1, m)$ is constructed by taking one correction term in the perturbation expansion and correction terms of $m$ th-order derivatives in the Taylor series expansion:

Consider the following system of first-order differential equations.

$$
F_{k}\left(\dot{u}_{k}, u_{j}, \varepsilon, t\right)=0, \quad k=1,2, \ldots, K, \quad j=1,2, \ldots, K
$$

where $K$ represents the number of differential equations in the system and the number of dependent variables. $K=1$ for a single equation. In the open form, the system of equations is

$$
\begin{gathered}
F_{1}=F_{1}\left(\dot{u}_{1}, u_{1}, u_{2}, \ldots u_{K}, \varepsilon, t\right)=0, \\
F_{2}=F_{2}\left(\dot{u}_{2}, u_{1}, u_{2}, \ldots u_{K}, \varepsilon, t\right)=0, \\
\vdots \\
F_{K}=F_{K}\left(\dot{u}_{K}, u_{1}, u_{2}, \ldots u_{K}, \varepsilon, t\right)=0 .
\end{gathered}
$$

Assume an approximate solution of the system

$$
u_{k, n+1}=u_{k, n}+\varepsilon u_{k, n}^{c}
$$

with one correction term in the perturbation expansion. The subscript $n$ represents the $n$th iteration over this approximate solution. The system can be approximated with a Taylor series expansion in the neighborhood of $\varepsilon=0$ as

$$
F_{k}=\sum_{m=0}^{M} \frac{1}{m !}\left[\left(\frac{d}{d \varepsilon}\right)^{m} F_{k}\right]_{\varepsilon=0} \varepsilon^{m}, \quad k=1,2, \ldots, K
$$

where

$$
\frac{d}{d \varepsilon}=\frac{\partial \dot{u}_{k, n+1}}{\partial \varepsilon} \frac{\partial}{\partial \dot{u}_{k, n+1}}+\sum_{j=1}^{K}\left(\frac{\partial u_{j, n+1}}{\partial \varepsilon} \frac{\partial}{\partial u_{j, n+1}}\right)+\frac{\partial}{\partial \varepsilon}
$$

is defined for the $n+1$ th iterative equation

$$
F_{k}\left(\dot{u}_{k, n+1}, u_{j, n+1}, \varepsilon, t\right)=0
$$

Substituting (5) into (4), one obtains an iteration equation

$$
\begin{gathered}
F_{k}=\sum_{m=0}^{M} \frac{1}{m !}\left[\left(\dot{u}_{k, n}^{c} \frac{\partial}{\partial \dot{u}_{k, n+1}}+\sum_{j=1}^{K} u_{j, n}^{c} \frac{\partial}{\partial u_{j, n+1}}+\frac{\partial}{\partial \varepsilon}\right)^{m} F_{k}\right]_{\varepsilon=0} \\
\times \varepsilon^{m}=0, \quad k=1,2 \ldots K
\end{gathered}
$$

which is a first-order differential equation and can be solved for the correction terms $u_{k, n}^{c}$. Then, using (3), the $n+1$ th iteration solution can be found. Iterations are terminated after a successful approximation is obtained.

Note that for a more general algorithm, $n$ correction terms instead of one can be taken in expansion (3) which would then be a $\operatorname{PIA}(n, m)$ algorithm. The algorithm can also be generalized to a differential equation system having arbitrary order of derivatives.

\section{Applications}

Applications of the theory developed will be outlined in this section. A first-order single equation and coupled systems with two and three equations will be treated.

Example 1. The following first-order differential equation arising in the cooling problem of a lumped system [21]

$$
(1+\varepsilon u) \frac{d u}{d t}+u=0, \quad u(0)=1
$$

will be treated with $\operatorname{PIA}(1,1)$ and $\operatorname{PIA}(1,2)$ algorithms. The specific heat is assumed to be a linear function of temperature and the equation is cast into nondimensional form as outlined in [21].

(i) $\operatorname{PIA}(1,1)$ Algorithm. For the equation considered, taking $M=1$ and $K=1,(7)$ reduces to

$$
\dot{u}_{1, n}^{c}+u_{1, n}^{c}=-\frac{\dot{u}_{1, n}+u_{1, n}}{\varepsilon}-u_{1, n} \dot{u}_{1, n}
$$

which is the determining iteration equation for the perturbation correction term. Assuming an initial solution, using then (9) and (3), successive iteration functions can be determined. An initial trial function

$$
u_{1,0}=e^{-t}
$$

which satisfies the boundary condition is selected. Substituting this trial function into (9), solving for the correction term, and using (3), one has

$$
u_{1,1}=(1+\varepsilon) e^{-t}-\varepsilon e^{-2 t}
$$


The successive iterations are

$$
\begin{aligned}
u_{1,2}= & \left(1+\varepsilon+\frac{\varepsilon^{2}}{2}+\frac{\varepsilon^{3}}{6}\right) e^{-t}-\varepsilon\left(1+2 \varepsilon+\varepsilon^{2}\right) e^{-2 t} \\
& +\frac{3}{2} \varepsilon^{2}(1+\varepsilon) e^{-3 t}-\frac{2}{3} \varepsilon^{3} e^{-4 t} \\
u_{1,3}= & \frac{e^{-t}}{840}\left(840+840 \varepsilon+420 \varepsilon^{2}+140 \varepsilon^{3}\right. \\
& -\frac{e^{-2 t}}{36} \varepsilon\left(6+6 \varepsilon+3 \varepsilon^{2}+\varepsilon^{3}\right)^{2} \\
& +\frac{e^{-3 t}}{4} \varepsilon^{2}(1+\varepsilon)^{2}\left(6+6 \varepsilon+3 \varepsilon^{2}+\varepsilon^{3}\right) \\
& -\frac{e^{-4 t}}{3} \varepsilon^{3}\left(8+20 \varepsilon+21 \varepsilon^{2}+12 \varepsilon^{3}+3 \varepsilon^{6}\right) \\
& +\frac{5 e^{-5 t}}{72} \varepsilon^{4}\left(39+93 \varepsilon+87 \varepsilon^{2}+29 \varepsilon^{3}\right) \\
& -\frac{43 e^{-6 t}}{20} \varepsilon^{5}(1+\varepsilon)^{2}+\frac{7 e^{-7 t}}{6} \varepsilon^{6}(1+\varepsilon)-\frac{16 e^{-8 t}}{63} \varepsilon^{7}
\end{aligned}
$$

These results are the same with the results of the variational iteration method given in [21].

(ii) PIA(1,2) Algorithm. A higher iteration algorithm can be constructed by taking $M=2$. For this choice, (7) reduces to

$$
\left(1+\varepsilon u_{1, n}\right) \dot{u}_{1, n}^{c}+\left(1+\varepsilon \dot{u}_{1, n}\right) u_{1, n}^{c}=-\frac{\dot{u}_{1, n}+u_{1, n}}{\varepsilon}-u_{1, n} \dot{u}_{1, n} .
$$

Taking the same initial trial function as given in (10), the successive iterations are

$$
\begin{aligned}
u_{1,1}= & e^{-t}+\frac{2 \varepsilon\left(e^{t}-1\right)+\varepsilon^{2}\left(e^{t}-e^{-t}\right)}{2\left(e^{t}+\varepsilon\right)^{2}}, \\
u_{1,2}= & e^{-t}+\frac{2 \varepsilon\left(e^{t}-1\right)+\varepsilon^{2}\left(e^{t}-e^{-t}\right)}{2\left(e^{t}+\varepsilon\right)^{2}} \\
& +\frac{\varepsilon^{3}}{24\left(e^{t}+\varepsilon\right)^{5}} e^{-t}\left(-1+e^{t}\right)^{2} \\
& \times\left(-16 e^{2 t}+4 e^{3 t}-15 e^{t} \varepsilon-10 e^{2 t} \varepsilon\right. \\
& \left.+e^{3 t} \varepsilon-3 \varepsilon^{2}-6 e^{t} \varepsilon^{2}-3 e^{2 t} \varepsilon^{2}\right) .
\end{aligned}
$$

In (13), during the iterations, the resulting equation comes out to be a variable coefficient system. For obtaining the last iteration, due to complexity, $u_{1,0}$ is taken instead of $u_{1,1}$ in the coefficients of the left-hand side. The third iteration result is not given here for brevity.
TABLE 1: Comparison of percentage errors of $\operatorname{PIA}(1,2)$ with $\operatorname{PIA}(1,1)$ and VIM for $\varepsilon=1$.

\begin{tabular}{lcccccc}
\hline \multirow{4}{*}{$t$} & \multicolumn{2}{c}{ \% error for PIA(1,2) } & \multicolumn{3}{c}{ error for PIA(1,1) and VIM } \\
& $u_{1}$ & $u_{2}$ & $u_{3}$ & $u_{1}$ & $u_{2}$ & $u_{3}$ \\
\hline 0 & 0.00 & 0.00 & 0.00 & 0.00 & 0.00 & 0.00 \\
1 & 1.77 & 0.19 & 0.02 & 5.87 & 1.71 & 3.15 \\
2 & 0.29 & 0.26 & 0.04 & 9.38 & 5.88 & 1.53 \\
3 & 3.98 & 0.30 & 0.00 & 19.11 & 2.66 & 0.63 \\
4 & 6.31 & 0.00 & 0.03 & 23.55 & 0.08 & 0.99 \\
5 & 7.36 & 0.21 & 0.03 & 25.34 & 1.12 & 0.97 \\
6 & 7.78 & 0.30 & 0.03 & 26.02 & 1.61 & 0.93 \\
7 & 7.94 & 0.34 & 0.02 & 26.28 & 1.79 & 0.92 \\
\hline \% mean & 4.55 & 0.20 & 0.02 & 17.86 & 2.58 & 1.40 \\
error & & & & & &
\end{tabular}

The error in Table 1 is defined as

$$
\begin{aligned}
\% \text { error }= & \frac{\mid \text { numerical solution }- \text { approximate solution } \mid}{\text { numerical solution }} \\
& \times 100 .
\end{aligned}
$$

As can be seen from Table $1, \operatorname{PIA}(1,2)$ performs better than VIM and PIA $(1,1)$.

Example 2. Two coupled stiff system will now be considered. Solutions will be obtained by $\operatorname{PIA}(1,1)$ algorithm. The coupled system is [22]

$$
\begin{gathered}
\dot{u}_{1}=-1002 u_{1}+1000 u_{2}^{2}, \\
\dot{u}_{2}=u_{1}-u_{2}-u_{2}^{2},
\end{gathered}
$$

with the initial conditions

$$
u_{1}(0)=1, \quad u_{2}(0)=1,
$$

for which exact solutions are available as

$$
u_{1}=e^{-2 t}, \quad u_{2}=e^{-t} .
$$

An artificial perturbation parameter is inserted as follows:

$$
\begin{gathered}
F_{1}=\dot{u}_{1, n+1}+1002 u_{1, n+1}-\varepsilon 1000 u_{2, n+1}^{2}=0, \\
F_{2}=\dot{u}_{2, n+1}+u_{2, n+1}-u_{1, n+1}+\varepsilon u_{2, n+1}^{2}=0 .
\end{gathered}
$$

For (19), (7) reduces to

$$
\begin{gathered}
\varepsilon \dot{u}_{1, n}^{c}+1002 \varepsilon u_{1, n}^{c}=-\dot{u}_{1, n}-1002 u_{1, n}+\varepsilon 1000 u_{2, n}^{2}, \\
\varepsilon \dot{u}_{2, n}^{c}+\varepsilon u_{2, n}^{c}=-\dot{u}_{2, n}-u_{2, n}-\varepsilon u_{2, n}^{2}+u_{1, n+1} .
\end{gathered}
$$

If the initial trial functions are taken as

$$
\begin{aligned}
& u_{1,0}=1, \\
& u_{2,0}=1,
\end{aligned}
$$




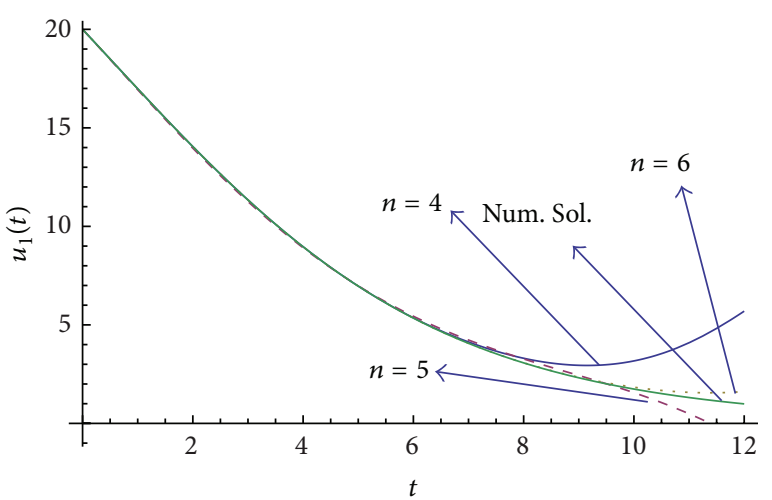

Figure 1: Comparison of iterations $(n=4,5,6)$ of PIA $(1,1)$ and numerical solutions for $u_{1}$.

the successive iterations are

$$
\begin{gathered}
u_{1,1}=\frac{500}{501}+\frac{e^{-1002 t}}{501}, \\
u_{2,1}=-\frac{1}{501}-\frac{e^{-1002 t}}{501501}+\frac{1003 e^{-t}}{1001}, \\
u_{1,2}=\frac{500}{125751501}-\frac{500 e^{-2004 t}}{126003129753501} \\
+\frac{2006000 e^{-1003 t}}{502002501}+\frac{1006009 e^{-2 t}}{1002001}-\frac{2006000 e^{-t}}{502002501} \\
+e^{-1002 t}\left(-\frac{1007012008}{251503253001}+\frac{2000 t}{251252001}\right), \\
u_{2,2}=-\frac{1}{125751501}+\frac{e^{-2004 t}}{252132136759503} \\
+\frac{1003 e^{-1003 t}}{251252001} \\
+e^{-1002 t}\left(\frac{335670670}{83918252084667}-\frac{2000 t}{251503253001}\right) \\
\left.+\frac{504264776770742984}{504264776776764003}+\frac{2006 t}{502002501}\right) .
\end{gathered}
$$

These results are identical with the results of the variational iteration method given in [22].

Example 3. The problem of spreading of a nonfatal disease in a population which is assumed to have constant size over the period of the epidemic is considered in [23]. The following system determines the progress of the disease:

$$
\begin{gathered}
\dot{u}_{1}=-\beta u_{1} u_{2}, \\
\dot{u}_{2}=\beta u_{1} u_{2}-\gamma u_{2}, \\
\dot{u}_{3}=\gamma u_{2}, \\
u_{1}(0)=20, \quad u_{2}(0)=15, \quad u_{3}(0)=10 .
\end{gathered}
$$

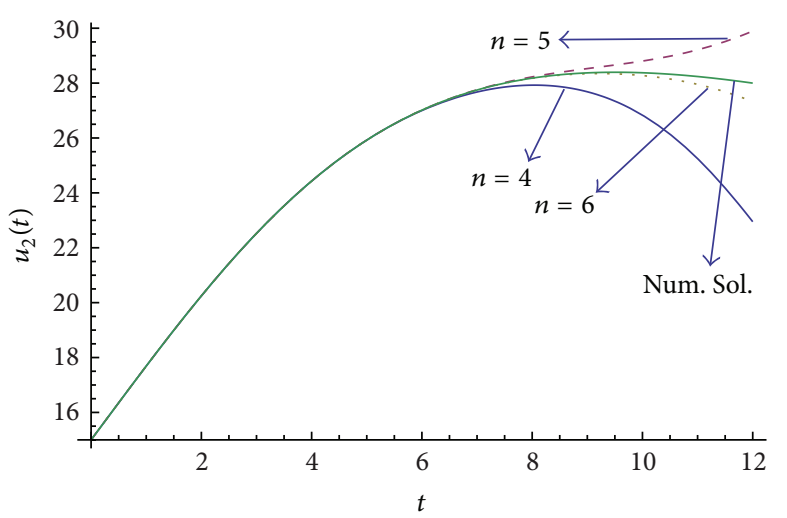

Figure 2: Comparison of iterations $(n=4,5,6)$ of PIA $(1,1)$ and numerical solutions for $u_{2}$.

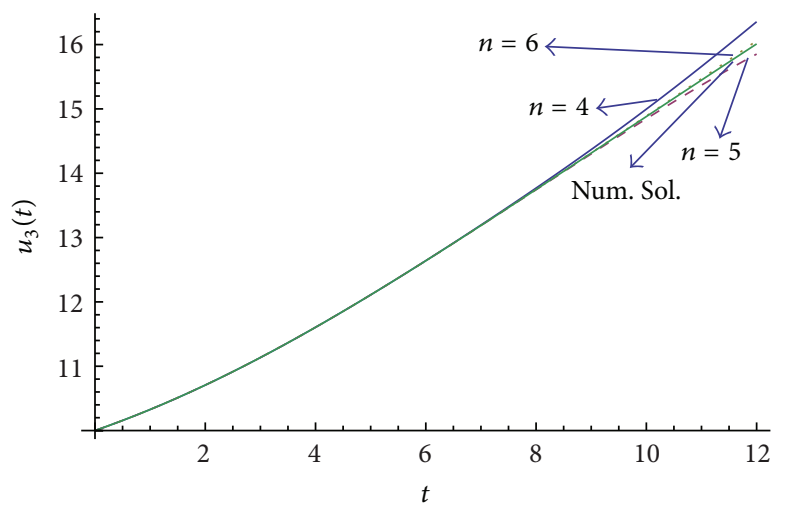

Figure 3: Comparison of iterations $(n=4,5,6)$ of PIA $(1,1)$ and numerical solutions for $u_{3}$.

The system is solved using PIA $(1,1)$. Perturbation parameter is artificially introduced as

$$
\begin{gathered}
F_{1}=\dot{u}_{1, n+1}+\beta \varepsilon u_{1, n+1} u_{2, n+1}=0, \\
F_{2}=\dot{u}_{2, n+1}-\beta \varepsilon u_{1, n+1} u_{2, n+1}+\gamma \varepsilon u_{2, n+1}=0, \\
F_{3}=\dot{u}_{3, n+1}-\gamma \varepsilon u_{2, n+1}=0 .
\end{gathered}
$$

For the previous equations, (7) reduces to

$$
\begin{gathered}
\dot{u}_{1, n}+\varepsilon \dot{u}_{1, n}^{c}+\beta \varepsilon u_{1, n} u_{2, n}=0, \\
\dot{u}_{2, n}+\varepsilon \dot{u}_{2, n}^{c}+\varepsilon\left(\gamma-\beta u_{1, n}\right) u_{2, n}=0, \\
\dot{u}_{3, n}+\varepsilon \dot{u}_{3, n}^{c}-\gamma \varepsilon u_{2, n}=0 .
\end{gathered}
$$

The initial trial functions are

$$
\begin{aligned}
& u_{1,0}=20, \\
& u_{2,0}=15, \\
& u_{3,0}=10 .
\end{aligned}
$$


The following iteration results are obtained for $\beta=1 / 100$ and $\gamma=1 / 50$ :

$$
\begin{aligned}
& u_{1,1}=20-3 t \\
& u_{2,1}=15+2.7 t \\
& u_{3,1}=10+0.3 t \\
& u_{1,2}=20-3 t-0.045 t^{2}+0.027 t^{3} \text {, } \\
& u_{2,2}=15+2.7 t+0.018 t^{2}-0.027 t^{3} \text {, } \\
& u_{3,2}=10+0.3 t+0.027 t^{2}, \\
& u_{1,3}=20-3 t-\frac{9}{2} \times 10^{-2} t^{2}+\frac{561}{2} \times 10^{-4} t^{3} \\
& -\frac{621}{8} \times 10^{-5} t^{4}-\frac{15309}{5} \times 10^{-7} t^{5} \\
& -\frac{567}{2} \times 10^{-8} t^{6}+\frac{729}{7} \times 10^{-8} t^{7} \\
& u_{2,3}=15+27 \times 10^{-2} t+\frac{9}{5} \times 10^{-2} t^{2} \\
& -2817 \times 10^{-5} t^{3}-\frac{513}{8} \times 10^{-5} t^{4}+\frac{15309}{5} \\
& \times 10^{-7} t^{5}+\frac{567}{2} \times 10^{-8} t^{6}-\frac{729}{7} \times 10^{-8} t^{7}, \\
& u_{3,3}=10+3 \times 10^{-1} t+27 \times 10^{-3} t^{2} \\
& +\frac{3}{25} \times 10^{-3} t^{3}-\frac{27}{2} \times 10^{-5} t^{4} \\
& u_{1,4}=20-3 t-\frac{9}{2} \times 10^{-2} t^{2}+\frac{561}{2} \times 10^{-4} t^{3} \\
& -\frac{6363}{8} \times 10^{-6} t^{4}-\frac{126603}{4} \times 10^{-8} t^{5}-\frac{97641}{2} \\
& \times 10^{-9} t^{6}+\frac{9913563}{28} \times 10^{-11} t^{7} \\
& +\frac{112714011}{112} \times 10^{-13} t^{8}-\frac{1398332889}{56} \times 10^{-15} t^{9} \\
& -\frac{13180077}{2} \times 10^{-16} t^{10}+\frac{943578963}{7} \times 10^{-18} t^{11} \\
& +\frac{334611}{125} \times 10^{-15} t^{12}-\frac{25187679}{52} \times 10^{-18} t^{13} \\
& +\frac{59049}{14} \times 10^{-18} t^{14}+\frac{177147}{245} \times 10^{-18} t^{15}, \\
& u_{2,4}=15+\frac{27}{10} t+\frac{9}{500} t^{2}-2817 \times 10^{-5} t^{3} \\
& -\frac{26181}{4} \times 10^{-7} t^{4}+\frac{127629}{4} \times 10^{-8} t^{5} \\
& +\frac{447381}{4} \times 10^{-10} t^{6}-\frac{9936243}{28} \times 10^{-11} t^{7} \\
& -\frac{102798011}{112} \times 10^{-13} t^{8}+\frac{1398332889}{56} \times 10^{-15} t^{9}
\end{aligned}
$$

$$
\begin{aligned}
+ & \frac{13180077}{2} \times 10^{-16} t^{10}-\frac{943578963}{7} \times 10^{-18} t^{11} \\
- & \frac{334611}{125} \times 10^{-15} t^{12}+\frac{25187679}{52} \times 10^{-18} t^{13} \\
+ & \frac{59049}{14} \times 10^{-18} t^{14}-\frac{177147}{245} \times 10^{-18} t^{15} \\
u_{3,4}= & 10+\frac{3}{10} t+\frac{27}{1000} t^{2}+\frac{3}{25000} t^{3}-\frac{2817}{2} \times 10^{-7} t^{4} \\
& -\frac{513}{5} \times 10^{-8} t^{5}+\frac{5103}{5} \times 10^{-9} t^{6} \\
& +81 \times 10^{-10} t^{7}-\frac{729}{28} \times 10^{-10} t^{8}
\end{aligned}
$$

The previous solutions are the same as those obtained from variational iteration method [23].

Functions $u_{1-3}$ are plotted in Figures 1, 2, and 3. The higher iterations, that is, $n=4,5,6$, calculated by symbolic programs are compared with the numerical solutions. As the number of iterations increase, the approximate analytical solutions converge to the numerical solutions.

\section{Concluding Remarks}

The newly developed perturbation-iteration algorithm is applied to systems of equations for the first time. The theory is developed first and then applied to three different problems. Based on this study and on the previous work [17], one can conclude that while $\operatorname{PIA}(1,1)$ algorithm produces compatible results with the VIM method, $\operatorname{PIA}(1,2)$ produces better results than the PIA $(1,1)$ and the VIM.

\section{References}

[1] A. H. Nayfeh, Perturbation Methods, Wiley-Interscience, New York, NY, USA, 1973.

[2] A. V. Skorokhod, F. C. Hoppensteadt, and H. Salehi, Random Perturbation Methods with Applications in Science and Engineering, Springer, New York, NY, USA, 2002.

[3] J.-H. He, "Iteration perturbation method for strongly nonlinear oscillations," Journal of Vibration and Control, vol. 7, no. 5, pp. 631-642, 2001.

[4] R. E. Mickens, "Iteration procedure for determining approximate solutions to nonlinear oscillator equations," Journal of Sound and Vibration, vol. 116, no. 1, pp. 185-187, 1987.

[5] R. E. Mickens, "A generalized iteration procedure for calculating approximations to periodic solutions of 'truly nonlinear oscillators," Journal of Sound and Vibration, vol. 287, no. 4-5, pp. 1045$1052,2005$.

[6] R. E. Mickens, "Iteration method solutions for conservative and limit-cycle force oscillators," Journal of Sound and Vibration, vol. 292, no. 3-5, pp. 964-968, 2006.

[7] K. Cooper and R. E. Mickens, "Generalized harmonic balance/numerical method for determining analytical approximations to the periodic solutions of the potential," Journal of Sound and Vibration, vol. 250, no. 5, pp. 951-954, 2002. 
[8] H. Hu and Z.-G. Xiong, "Oscillations in an $x^{(2 m+1) /(2 n+1)}$ potential," Journal of Sound and Vibration, vol. 259, no. 4, pp. 977-980, 2003.

[9] M. A. Abdou, "On the variational iteration method," Physics Letters A, vol. 366, no. 1-2, pp. 61-68, 2007.

[10] S. Q. Wang and J. H. He, "Nonlinear oscillator with discontinuity by parameter-expansion method," Chaos, Solitons and Fractals, vol. 35, no. 4, pp. 688-691, 2008.

[11] J.-H. He, "Modified Lindstedt-Poincaré methods for some strongly non-linear oscillations, part I: expansion of a constant," International Journal of Non-Linear Mechanics, vol. 37, no. 2, pp. 309-314, 2002.

[12] J. I. Ramos, "On Linstedt-Poincaré technique for the quintic Duffing equation," Applied Mathematics and Computation, vol. 193, no. 2, pp. 303-310, 2007.

[13] T. Öziş and A. Yıldırım, "Determination of periodic solution for a $u^{1 / 3}$ force by He's modified Lindstedt-Poincaré method," Journal of Sound and Vibration, vol. 301, no. 1-2, pp. 415-419, 2007.

[14] R. E. Mickens, "Oscillations in an $x^{4 \times 3}$ potential," Journal of Sound and Vibration, vol. 246, no. 2, pp. 375-378, 2001.

[15] M. Pakdemirli, Y. Aksoy, and H. Boyac1, "A new perturbationiteration approach for first order differential equations," Mathematical and Computational Applications, vol. 16, no. 4, pp. 890899, 2011.

[16] Y. Aksoy and M. Pakdemirli, "New perturbation-iteration solutions for Bratu-type equations," Computers and Mathematics with Applications, vol. 59, no. 8, pp. 2802-2808, 2010.

[17] Y. Aksoy, M. Pakdemirli, and S. Abbasbandy, "New perturbation-iteration solutions for nonlinear heat transfer equations," International Journal of Numerical Methods for Heat and Fluid Flow, vol. 22, no. 7, pp. 814-828, 2012.

[18] M. Pakdemirli and H. Boyac1, "Generation of root finding algorithms via perturbation theory and some formulas," Applied Mathematics and Computation, vol. 184, no. 2, pp. 783-788, 2007.

[19] M. Pakdemirli, H. Boyac1, and H. A. Yurtsever, "Perturbative derivation and comparisons of root-finding algorithms with fourth order derivatives," Mathematical and Computational Applications, vol. 12, no. 2, pp. 117-124, 2007.

[20] M. Pakdemirli, H. Boyac1, and H. A. Yurtsever, "A rootfinding algorithm with fifth order derivatives," Mathematical and Computational Applications, vol. 13, no. 2, pp. 123-128, 2008.

[21] H. Tari, D. D. Ganji, and H. Babazadeh, “The application of He's variational iteration method to nonlinear equations arising in heat transfer," Physics Letters A, vol. 363, no. 3, pp. 213-217, 2007.

[22] M. T. Darvishi, F. Khani, and A. A. Soliman, "The numerical simulation for stiff systems of ordinary differential equations," Computers and Mathematics with Applications, vol. 54, no. 7-8, pp. 1055-1063, 2007.

[23] M. Rafei, H. Daniali, and D. D. Ganji, "Variational interation method for solving the epidemic model and the prey and predator problem," Applied Mathematics and Computation, vol. 186, no. 2, pp. 1701-1709, 2007. 


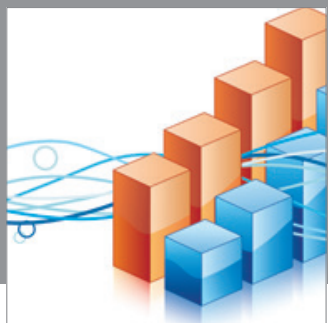

Advances in

Operations Research

mansans

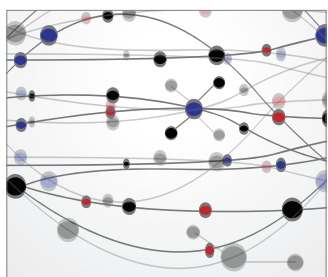

The Scientific World Journal
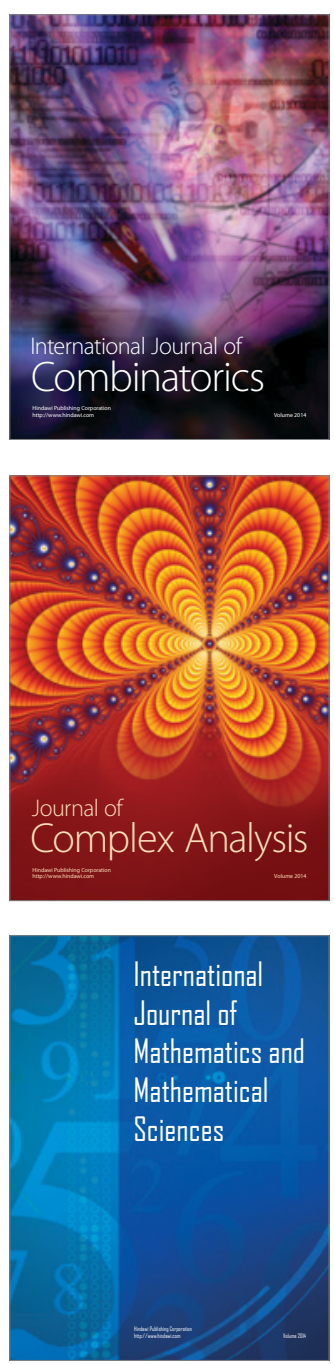
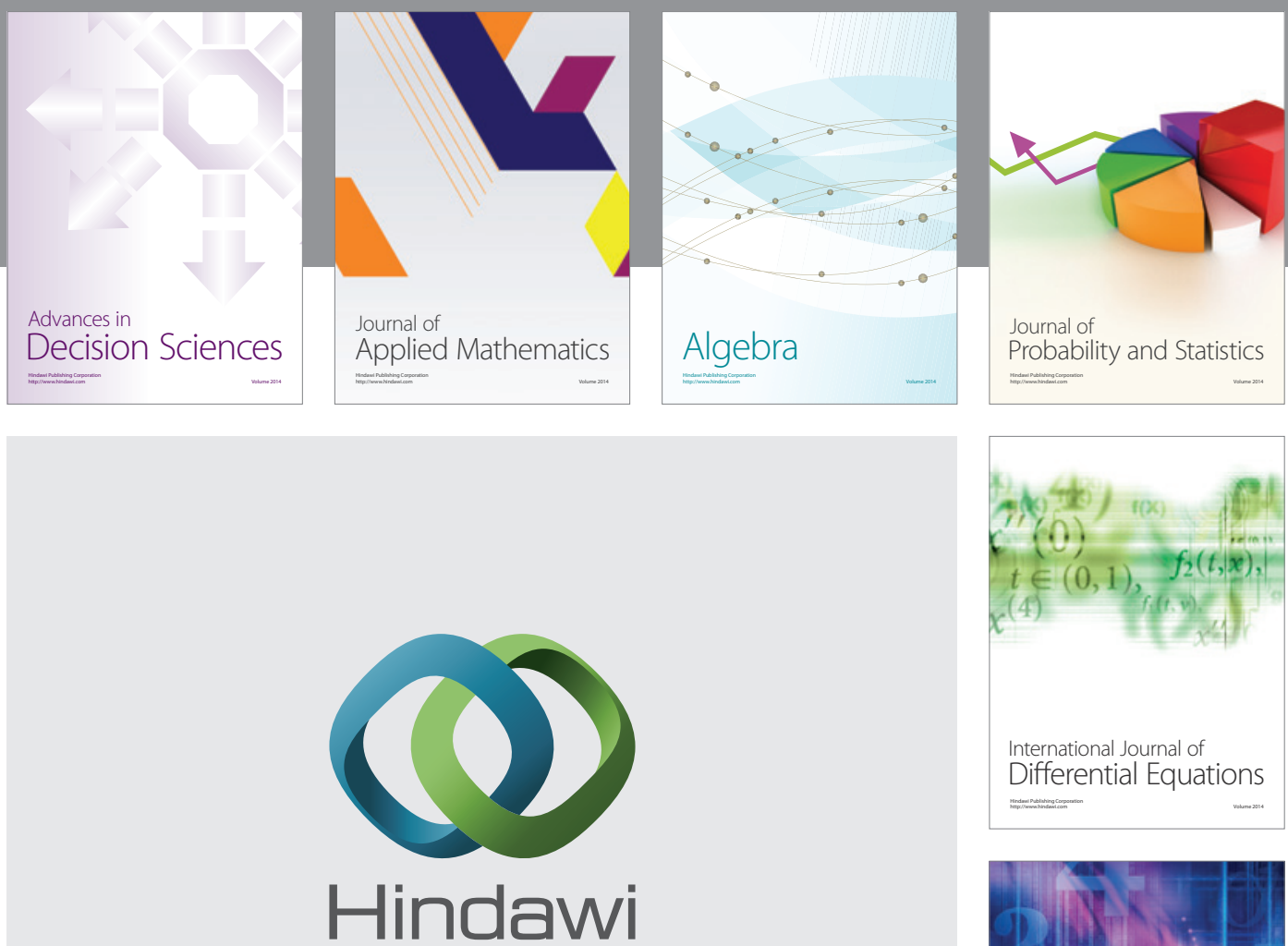

Submit your manuscripts at http://www.hindawi.com
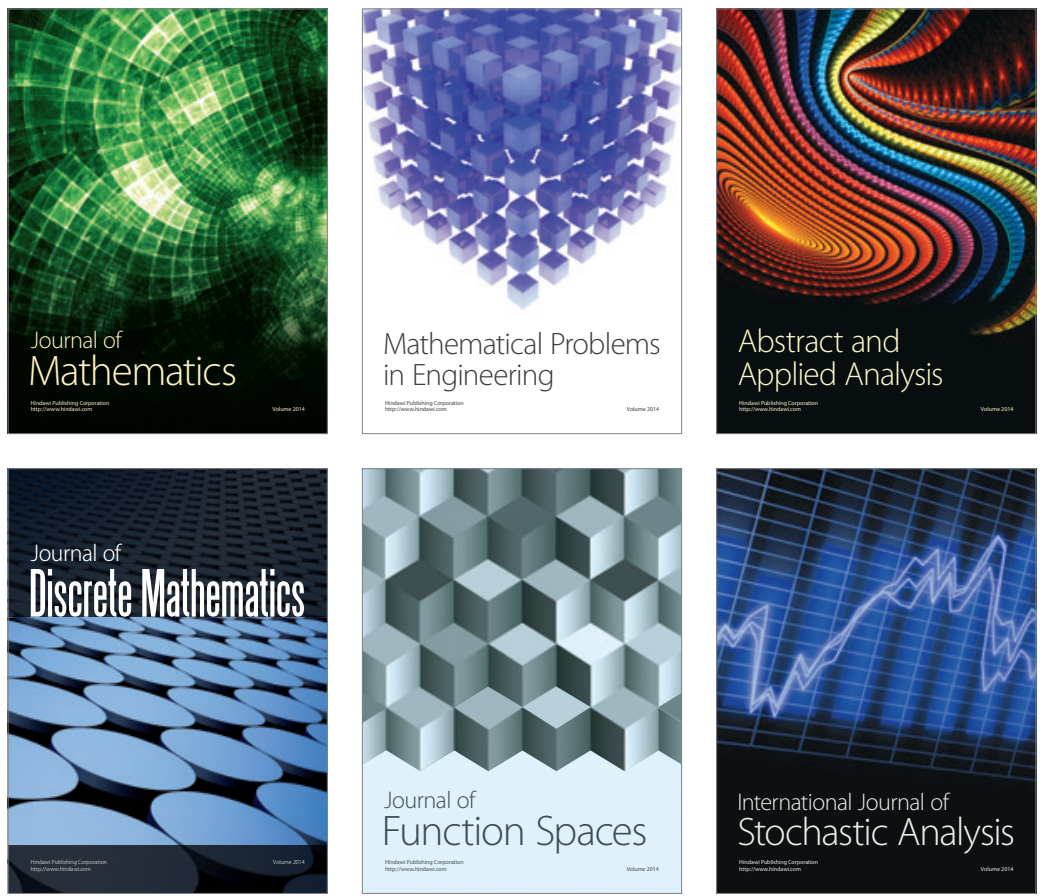

Journal of

Function Spaces

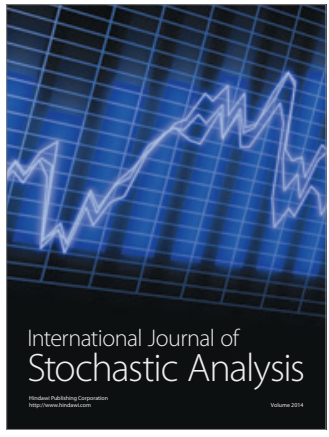

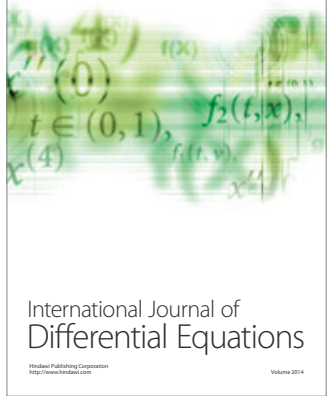
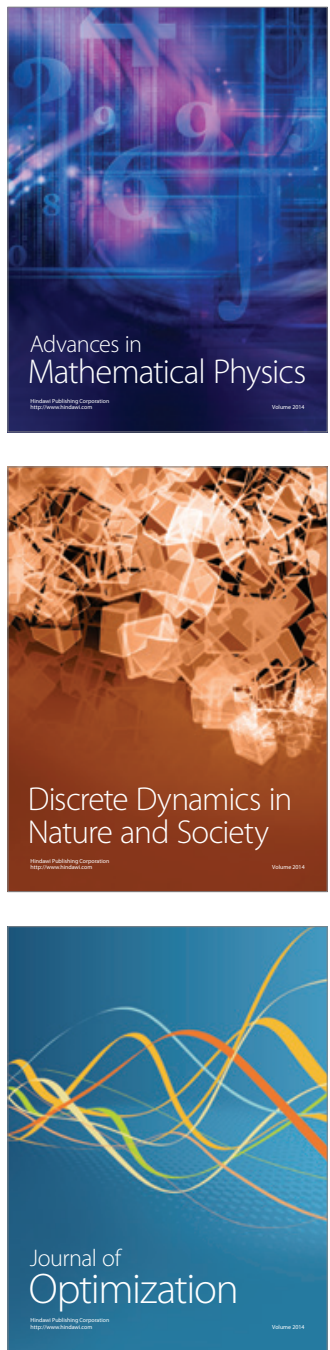